\title{
S-Methylene linkage comprising 1,3,4-oxadiazoles: synthesis, reaction optimization and in vitro anti-microbial potential
}

\author{
Gautam M. Dhuda ${ }^{a}$, Khushal M. Kapadiya ${ }^{b}$, Paresh D. Ladwa ${ }^{a}$ and Jayesh J. Modha ${ }^{a^{*}}$
}

${ }^{a}$ Maharshi Dayanand Science College, Department of Chemistry, Bhaktakavi Narsinh Mehta University, Porbandar, Gujarat, India ${ }^{b}$ School of Science, Department of Chemistry, BRCC Laboratory, RK University, Rajkot, Gujarat, India

\begin{tabular}{l} 
C H R O N I C L E \\
\hline Article history: \\
Received July 1,2020 \\
Received in revised form \\
October 26, 2020 \\
Accepted October 31, 2020 \\
Available online \\
November 2, 2020 \\
\hline Keywords: \\
1,3,4-Oxadiazoles \\
Anti-microbial study \\
Copper catalyst \\
S-linkage chemistry \\
Synthetic optimization \\
\end{tabular}

\begin{abstract}
A B S T R A C T
To generate a new class of scaffolds with amended anti-microbial potency, synthesis and in vitro biological evaluation of a series of 2-((5-(3-nitrophenyl)-1,3,4-oxadiazol-2yl)methylthio)-5-substitutedphenyl-1,3,4-oxadiazole (5a-5o) having S-Methylene linkage between the two 1,3,4-diisoxazole rings is reported. Anti-microbial properties of the entire compounds were investigated in a broad panel of some selected gram-positive bacterial strains, gram-negative bacterial strain, and fungal strains using the broth microdilution method. The results were dominant on $\mathbf{- C l}$ and $-\mathbf{N} \mathbf{O}_{2}$ functionality than the used standard drug.
\end{abstract}

\section{Introduction}

Despite a large number of antibiotics and chemotherapeutics available for medical use, the antimicrobial resistance created a substantial need for a new class of anti-microbial Agents in the last decades ${ }^{1}$. A literature survey revealed that substances like 1,3,4-oxadiazole ${ }^{2}, 1,3,4-$ thiadiazole $^{3}$, and 1,2,4-triazole ${ }^{4}$ moieties had occupied a unique position in the design and synthesis of biologically active agents with remarkable anti-bacterial, analgesic, and anti-inflammatory activities. Out of various isomeric form, the existence of the 1,3,4-oxadiazole ring affects the physicochemical and pharmacokinetic properties of the compound and show better metabolic stability, water solubility, and lower lipophilicity. The various application of the 1,3,4-oxadiazoles ring in the therapeutic area can be listed as anticoagulant ${ }^{5}$, antibacterial ${ }^{6}$, antifungal ${ }^{7}$, anticancer ${ }^{8}$, anti-inflammatory ${ }^{9}$, insecticidal $^{10}$, antihypertensive ${ }^{11}$, etc. ${ }^{12}$.

Many routes of synthesis for the 1,3,4-oxadiazoles have been identified ${ }^{13-15}$ but, significantly fewer efforts have been made by the chemist to generate the ring using the metal-catalyzed process and environmentally fewer hazards recoverable catalysts. Moreover, one of the significant challenges in developing safe and effective antibody-drug conjugates (ADC) has been the generation of suitable chemical linkers between the drug and the antibody, but the linker chemistry is tricky. That's why less

* Corresponding author. Tel.: +91 9586980779

E-mail address: Khushal kapadiya06@yahoo.com (J. J. Modha)

(C) 2021 Growing Science Ltd. All rights reserved.

doi: $10.5267 /$ j.ccl.2020.11.001 
use is globally, and the S-methylene linker plays a vital role in the cytotoxic study. It was well studies that the oxadiazole ring connected via ester or amide linker exhibit promising biological efficacy against various cancer cell-lines and recently reported that the presence of a five-methylene linker turned out to be more effective for enhancing its effieciency ${ }^{16}$. Zhao et al. reported the evidence of small group linkers, e.g., methylene shows a significant role in strengthening the medicinal importance by which connect the anionic groups and aromatic groups to reduces the repulsive interaction ${ }^{17}$. Contour maps analyses indicated the importance of the linker between two aryl groups and the subtle differences of substituent groups ${ }^{18}$. Thus, the development of facile and efficient approaches to access these $1,3,4$-oxadiazoles is highly valuable to early drug discovery ${ }^{19-22}$.

During designing efficient bio-active molecules, a new concept is used. Two active pharmacophores are coupled to generate one novel structure having unpredictable anti-microbial activity than any of the used pharmacophore. Therefore, these observations prompted us to connected two 1,3,4-oxadiazole rings having $\mathrm{S}-\mathrm{CH}_{2}$ - linkage to enhance its potency. The synthesized diverse molecules were tested for their anti-microbial strength using some Gram (+ve) bacteria, Gram (-ve) bacteria, and some fungi strains.

\section{Results and Discussion}

\subsection{Chemistry and Spectroscopic discussion}

The multistep reaction sequences for the synthesis of the objective compounds $\mathbf{5 a - 5 0}$ are framed in Reaction scheme 1.

Reaction Scheme 1. Synthetic scheme of 2-((5-(3-nitrophenyl)-1,3,4-oxadiazol-2-yl)methylthio)-5substitutedphenyl-1,3,4-oxadiazole (5a-5o)
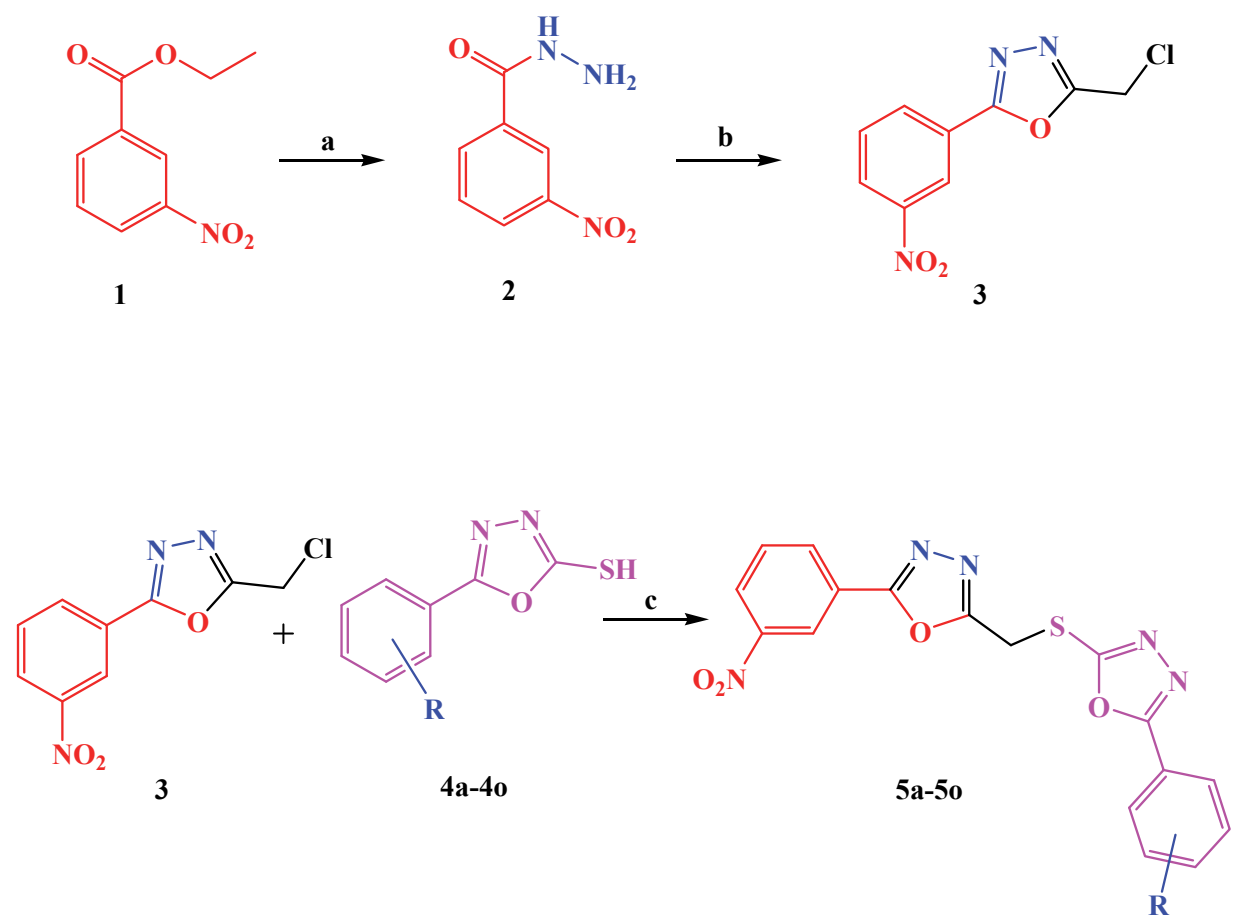

Reaction Conditions: (a) Et-OH/ $\mathrm{NH}_{2} \mathrm{NH}_{2}$, Reflux, 7h; (b) $\mathrm{POCl}_{3} / \mathrm{C}_{2} \mathrm{O}_{2} \mathrm{H}_{3} \mathrm{Cl}$, Reflux, 6h(c) CuI, Et $3 \mathrm{~N}, \mathrm{THF}$, RT.

3-Nitrobenzohydrazide was synthesized by the reaction of 3-nitro acid ester and hydrazine hydrazide under reflux conditions followed by $\mathrm{POCl}_{3}$ based cyclization to afford 2-(chloromethyl)-5(3-nitrophenyl)-1,3,4-oxadiazole (3) (92-97\%). 5-Phenyl-1,3,4-oxadiazole-2-thiols (4a-4o) with variant substitution, commercially available, were synthesized as the previously reported process. The final adduct 2-(3-nitrophenyl)-5-(((5-phenyl-1,3,4-oxadiazol-2-yl)thio)methyl)-1,3,4-oxadiazole (5a5o) were in turn prepared by soft metal $(\mathrm{CuI})$ catalyzed reaction between intermediate three and $4 \mathrm{a}-4 \mathrm{o}$ 
under primary conditions (TEA) gave "S-methylene linkage" containing novel product. It was found the analytically pure product $(75-93 \%)^{23}$.

As seen in Table 1, entry 1 is a traditional method, but it is suffering from low yield and also more consumption of time. But entry 2 shows the more product and less reaction time for the completion (1.5-2.0 Hours) due to a soft catalyst (CuI) with the best nucleophilic properties of iodine. While in the case of entry 3 and 4, the progress of the reaction was not observed in sodium iodide media and less yield in the case of $\mathrm{CuBr} / \mathrm{TEA}$ reaction conditions, respectively ${ }^{23,24}$.

Table 1. Reaction optimization in metal and non-metal catalyzed, and different alkaline media conditions

\begin{tabular}{ccccc}
\hline Entry & Compound Id & Catalyst & Base & Yield / Time \\
\hline $\mathbf{1}$ & & None & KOH/NaOH & $60-70 \% / 7-8$ hours \\
$\mathbf{2}$ & & CuI & TEA & KOH-95\%/ 1.5-2.0 hours \\
$\mathbf{3}$ & $\mathbf{5 a}$ & $\mathrm{NaI}$ & $\mathrm{KEH}$ & $-^{\mathrm{a}}$ \\
$\mathbf{4}$ & & $\mathrm{CuBr}$ & $\mathrm{b}$ & - \\
\hline
\end{tabular}

${ }^{a}$ reaction not proceeded; ${ }^{b}$ Minute quantity of yield (21\%).

Spectral data, i.e., ${ }^{1} \mathrm{H}$ NMR, ${ }^{13} \mathrm{C}$ NMR, IR, and Mass of all the synthesized compounds, were recorded and found in full agreement with the proposed structures.

On a close look at the spectral data, IR spectral data obtained here lead to the approval of the establishment of targeted compounds. The IR spectral data obtained for the final derivatives 5a-5o were suitable to a great extent in confirming their structure. Let us observe the spectral details of compound 5d and correlate the frequencies with the functional group present in the structure. Absorption peaks at 1026 and $1350 \mathrm{~cm}^{-1}$ helped confirm the presence of $-\mathrm{C}-\mathrm{O}-\mathrm{C}$ - in the oxadiazole nucleus. The absorption band at $1735 \mathrm{~cm}^{-1}$ proved the presence of $-\mathrm{C}=\mathrm{C}$ - in the aromatic ring. The $-\mathrm{NO}_{2}$ functional group (nitro) was also confirmed by a two sharp peak in 1473 and $1527 \mathrm{~cm}^{-1}$. An intense absorption band at 2931 $\mathrm{cm}^{-1}$ helped assign the presence of $-\mathrm{CH}_{2}$ (methylene group), and aromatic $-\mathrm{C}-\mathrm{H}$ was noticed at 3078 $\mathrm{cm}^{-1}$ in the compound 5d. A sharp absorption peak at $717 \mathrm{~cm}^{-1}$ helped to assign the presence of the C$\mathrm{Cl}$ functional group.

The ${ }^{1} \mathrm{H}$ Nuclear Magnetic Resonance study was conducted for each of the derivatives, and the $\delta$ values were recorded. The $\delta$ values were recorded corresponding to the methylene group and the protons of the aromatic ring. For compound $\mathbf{5 d}, \delta$ value at 5.03 as a singlet proved the presence of two hydrogen atoms of the methylene group. A multiplet obtained at 7.53 to $8.64 \mathrm{ppm}$ confirmed the presence of aromatic protons. ${ }^{13} \mathrm{C}$ NMR spectra exhibited tight singlet at $24 \mathrm{sppm}$ value, which showed the methylene bridge in the oxadiazole ring. The remaining aromatic carbons were in fair agreement with the theoretical values.

In mass spectra, the molecular ion peak observed at $\mathrm{m} / \mathrm{z} 404.3$ in $\mathbf{5 d}$ confirmed the formation of the desired structure.

\subsection{Biological Evaluation}

An anti-microbial evaluation was carried out at the Department of Microbiology, RK University, Rajkot. The activity was expressed in the dose-response assay (MIC) against the affected organism using five various strains using ciprofloxacin as the reference anti-bacterial agent. The anti-fungal activity was carried out using two selected fungal strains using fluconazole as a reference drug. Results were expressed in minimum inhibition concentration (MIC). The detailed protocol, as stated in our last published paper, was repeated over here ${ }^{25}$.

\subsubsection{Anti-microbial evaluation}

The newly synthesized entities (5a-5o) were screened for their anti-microbial assay against a broad panel of gram-positive bacteria $S$. aureus (ATCC No. 25923), E. faecalis (ATCC No. 29212), gramnegative bacteria E. coli (ATCC No. 25922), P. aeruginosa (ATCC No. 27853) and fungi C. Albicans 
(ATCC No. 10231), A. niger (ATCC No. 1015). Broad panel of gram-positive bacteria (S. aureus and E. faecalis), gram-negative bacteria (E. coli and P. aeruginosa), and fungal strains (C. Albicans and A. niger) were utilized for testing the anti-microbial properties of the targeted compounds (5a-5o). The anti-bacterial and anti-fungal activities in MIC $(\mu \mathrm{g} / \mathrm{ml})$ have been reported in Table 2. It was observed that most of the compounds exhibited excellent activity compared to the standards used, and very few of them exhibited moderate or low activity. The MIC value of ciprofloxacin was recorded to be $62.5 \mu \mathrm{g} / \mathrm{ml}$ against gram-positive bacterial strain $S$. aureus. The same standard responded at $125 \mu \mathrm{g} / \mathrm{ml}$ against $E$. faecalis, E. coli, and $P$. aeruginosa. Fluconazole, when used as a standard drug against fungal strains $C$. Albicans and A. niger, MIC value was observed at $125 \mu \mathrm{g} / \mathrm{ml}$ and $62.5 \mu \mathrm{g} / \mathrm{ml}$, respectively. In general, it was observed that most of the compounds have resulted in being potent anti-bacterial, and very few of them were good anti-fungal.

Table 2. Anti-microbial screening of the synthesized bis-1,3,4-oxadiazoles (5a-5o)

\begin{tabular}{|c|c|c|c|c|c|c|}
\hline \multirow{3}{*}{ Compounds } & \multicolumn{6}{|c|}{ Minimum Inhibitory Concentration (MIC) in $\mu \mathrm{g} / \mathrm{ml}$} \\
\hline & \multicolumn{2}{|c|}{ Gram-positive bacteria } & \multicolumn{2}{|c|}{ Gram-negative bacteria } & \multicolumn{2}{|c|}{ Fungi } \\
\hline & $\begin{array}{c}\text { S. aureus } \\
\text { ATCC } 25923\end{array}$ & $\begin{array}{c}\text { E. faecalis } \\
\text { ATCC } 29212\end{array}$ & $\begin{array}{c}\boldsymbol{E} . \text { coli } \\
\text { ATCC } 25922\end{array}$ & $\begin{array}{c}\text { P. aeruginosa } \\
\text { ATCC } 27853\end{array}$ & $\begin{array}{c}\text { C. albicans } \\
\text { ATCC } 10231\end{array}$ & $\begin{array}{c}\text { A. niger } \\
\text { ATCC } 1015\end{array}$ \\
\hline 5a & 125 & 125 & 125 & 125 & 125 & 62.5 \\
\hline $\mathbf{5 b}$ & 62.5 & 125 & 62.5 & 62.5 & 250 & 62.5 \\
\hline $5 c$ & 62.5 & 62.5 & 31.25 & 125 & 125 & 62.5 \\
\hline 5d & 31.25 & 62.5 & 31.25 & 31.25 & 250 & 31.25 \\
\hline $5 e$ & 62.5 & 62.5 & 15.62 & 31.25 & 125 & 31.25 \\
\hline $\mathbf{5 f}$ & 250 & 125 & 125 & 250 & 250 & 125 \\
\hline $5 g$ & 125 & 250 & 250 & 125 & 500 & 250 \\
\hline $5 \mathrm{~h}$ & 250 & 250 & 125 & 125 & 250 & 125 \\
\hline $5 \mathbf{i}$ & 62.5 & 125 & 250 & 125 & 250 & 250 \\
\hline $5 \mathbf{j}$ & 125 & 125 & 250 & 125 & 500 & 500 \\
\hline $5 \mathbf{k}$ & 62.5 & 125 & 62.5 & 62.5 & 250 & 250 \\
\hline 51 & 62.5 & 62.5 & 31.25 & 125 & 125 & 250 \\
\hline $5 \mathrm{~m}$ & 125 & 250 & 250 & 125 & 500 & 250 \\
\hline $5 n$ & 250 & 250 & 125 & 125 & 250 & 125 \\
\hline 50 & 62.5 & 62.5 & 15.62 & 31.25 & 125 & 31.25 \\
\hline Fluconazole & - & - & - & - & 125 & 62.5 \\
\hline Ciprofloxacin & 62.5 & 125 & 125 & 125 & - & - \\
\hline
\end{tabular}

MIC*: Minimum Inhibitory Concentration

\subsubsection{Anti-bacterial activity}

The anti-bacterial potential of the synthesized compounds (5a-5o) was tested against gram-positive bacteria $S$. aureus (ATCC no. 25923) and E. faecalis (ATCC no. 27853) and gram-negative bacteria $E$. coli (ATCC no. 25922) and $P$. aeruginosa (ATCC no. 27853). Compounds 5b (3-NO 2$), 5 \mathrm{c}\left(4-\mathrm{NO}_{2}\right), 5 \mathrm{~d}$ $(2-\mathrm{Cl}), 5 \mathrm{e}(4-\mathrm{Cl}), 5 \mathrm{k}(-4-\mathrm{Br}), 5 \mathrm{l}(-3,4-\mathrm{diCl})$, and $5 \mathrm{o}\left(-2,4-\mathrm{di}-\mathrm{NO}_{2}\right)$ were found to exhibit excellent or equivalent activity as compared to the standard ciprofloxacin. Other compounds $5 \mathrm{a}(\mathrm{H}), 5 \mathrm{~g}(2-\mathrm{I}), 5 \mathrm{i}(2-$ $\left.\mathrm{CH}_{3}\right), 5 \mathrm{j}\left(4-\mathrm{CH}_{3}\right), 5 \mathrm{~m}(-3,4-\mathrm{di}-\mathrm{OH})$, and $5 \mathrm{n}\left(-4-\mathrm{CHF}_{2}\right)$ were found to possess lower activity as compared to the standard drug and even poor than the formerly mentioned derivatives. When the same set of products 5a to 5o were tested against another gram-positive bacterial strain E. faecalis, all the derivatives other than compound $5 \mathrm{c}\left(4-\mathrm{NO}_{2}\right), 5 \mathrm{~d}(2-\mathrm{Cl})$, and $5 \mathrm{e}(4-\mathrm{Cl})$ resulted in equivalent or excellent activity. Compounds were exposed to gram-negative bacteria E. coli, and $5 \mathrm{e}$ was found to show perfect activity with MIC value $15.62 \mu \mathrm{g} / \mathrm{ml}$. Compounds $5 \mathrm{c}\left(4-\mathrm{NO}_{2}\right), 5 \mathrm{~d}(2-\mathrm{Cl})$, and 5o (-2,4-di-NO $\left.\mathrm{N}_{2}\right)$ were exhibiting $31.25 \mu \mathrm{g} / \mathrm{ml}$ MIC values, indicating better anti-bacterial potential than the standard ciprofloxacin. When the synthesized derivatives 5a to 50 were tested against gram-negative bacteria $P$. aeurginosa, it was found that compound $5 \mathrm{~b}(2-\mathrm{Cl}), 5 \mathrm{e}(4-\mathrm{Cl})$, and $5 \mathrm{o}\left(-2,4-\mathrm{di}_{-} \mathrm{NO}_{2}\right)$ exhibited excellent activity $(31.25 \mu \mathrm{g} / \mathrm{ml})$. The only derivative possessing insufficient activity than standard drug was compound $5 \mathrm{f}(2,4-\mathrm{diCl})$ against the gram-negative bacterial strain $P$. aeruginosa.

\subsubsection{Anti-fungal activity}

The anti-fungal tests were performed against two different fungal strains, A. niger, and C. Albicans, where fluconazole was used as a standard drug. The anti-fungal property of the synthesized derivatives (5a-5o) was found to be more effective on A. niger as compared to C. Albicans. On testing the 
compounds against $C$. Albicans, synthesized derivative $5 \mathrm{~d}(2-\mathrm{Cl})$ was found to show excellent activity compared to the standard drug fluconazole. Few other derivatives $5 \mathrm{a}(\mathrm{H}), 5 \mathrm{c}\left(4-\mathrm{NO}_{2}\right), 5 \mathrm{e}(4-\mathrm{Cl}), 51(-$ $3,4-\mathrm{diCl})$, and $50\left(-2,4-\mathrm{di}-\mathrm{NO}_{2}\right)$ were found to possess activity equivalent to that of the standard drug, while others were found to be low inactivity. The same derivatives, when tested for their anti-fungal property against a different fungal strain, $A$. niger, resulted in comparatively much better results than the former (C. Albicans). Compounds $5 \mathrm{~d}, 5 \mathrm{e}$, and 50 possessing $-\mathrm{Cl} /$-dinitro as a substituent exhibited excellent activity $(31.25 \mu \mathrm{g} / \mathrm{ml})$, even better than the standard drug fluconazole. Also, the derivatives possessing $-\mathrm{NO}_{2}$ were found to have equivalent activity $(62.5 \mu \mathrm{g} / \mathrm{ml})$ to that of the standard fluconazole. Products were including $-\mathrm{CH}_{3}$ as a functional group was found to show low activity (250 and 500 $\mu \mathrm{g} / \mathrm{ml})$ compared to standard drug.

\section{Conclusions}

In short, the idea of this report summarized that the compounds possessing electron-withdrawing substituents were found to exhibit excellent anti-bacterial and anti-fungal properties. Several derivatives were also showing even better results than the standard drug ciprofloxacin viz. compound 5e (4-Cl) showed MIC value even higher than standard drug against all bacterial and fungal strains $(15.62-62.5 \mu \mathrm{g} / \mathrm{ml})$ and the compound containing $-\mathrm{NO}_{2}$ and $-\mathrm{Cl}$ functional group was found to exhibit excellent property against gram-negative and gram-positive bacteria.

\section{Acknowledgment}

The authors gratefully acknowledge Maharshi Dayanand Science College, Porbandar, and School of Science, RK University, Rajkot, India, for laboratory and financial support.

\section{Experimental}

\subsection{Materials}

Chemicals and solvents were purchased from the Sigma-Aldrich Chemical Co., Merck chemical, Finar, and Spectrochem Ltd. All the chemicals were used without further purification. Thin-layer chromatography was accomplished on $0.2 \mathrm{~mm}$ precoated plates of Silica gel G60 F254 (Merck). Visualization was made under UV light (254 and $365 \mathrm{~nm}$ ). IR spectra were recorded on an "IR Affinity1S spectrophotometer (Shimadzu)" [DRS method, SN ratio: 30,000:1, maximum resolution of $0.5 \mathrm{~cm}^{-}$ , Optional extended wavenumber range: 25000 to $10 \mathrm{~cm}^{-1}$; Standard wave number measurement range: 7800 to $350 \mathrm{~cm}^{-1}$ ]. ${ }^{1} \mathrm{H}(400 \mathrm{MHz})$ and ${ }^{13} \mathrm{C}(101.1 \mathrm{MHz})$ NMR spectra were recorded on a "Bruker AVANCE II spectrometer" in DMSO-d6. Chemical shifts are expressed in $\delta$ ppm downfield from TMS. Mass spectra were determined by direct inlet probe on a "GC-MS-QP [Agilent 7820A-5977B; Ionization source: EI- $0.7 \mathrm{kV}$; Ion source temperature: $220{ }^{\circ} \mathrm{C}$; Interface temperature: $240{ }^{\circ} \mathrm{C}$ ] mass spectrometer". Solvents were evaporated with a "Roteva rotary evaporator." Melting points were measured in open capillaries and are uncorrected.

\subsection{Method of Synthesis}

\subsubsection{Procedure for the synthesis of 3-nitrobenzohydrazide (2).}

Dissolved $10 \mathrm{gm}(0.082 \mathrm{~mol})$ of ethyl 3-nitrobenzoate (1) in ethanol in a $100 \mathrm{ml}$ clean, dry round bottom flask under heating condition. To this reaction mixture, 0.041 moles of hydrazine hydrate was added and was refluxed for $7 \mathrm{hrs}$ at $70-80{ }^{\circ} \mathrm{C}$ temperature. Completion of the reaction was identified using Thin Layer Chromatography (TLC) using n-hexane: ethyl acetate $(8: 2 \mathrm{ml})$ as a mobile phase. After completing the reaction, it was poured into ice crushed water and stirred at a cooling temperature for 30 minutes. The solid separated was filtered off using wattmann filter paper and washed twice with the chilled water. The isolated powder material was crystalized using methanol to obtained an analytically pure product. 


\subsubsection{Procedure for the synthesis of 2-(chloromethyl)-5-(3-nitrophenyl)-1,3,4-oxadiazole (3).}

A vacuum dryer $100 \mathrm{ml} \mathrm{RBF}$, added $21 \mathrm{ml} \mathrm{POCl}_{3}, 0.049$ mole chloroacetic acid, and 0.017 moles of previously prepared intermediate (2) (Acid hydrazide). The reaction mixture was refluxed for $6 \mathrm{hrs}$ at $70-75{ }^{\circ} \mathrm{C}$ temperature. TLC was used to identify the completion of the reaction [Mobile phase: $\mathrm{n}-$ hexane: ethyl acetate $(8: 2 \mathrm{ml})]$. After completing the reaction, it was poured into ice crushed water and stirred at a cooling temperature for 15 minutes. The solid separated was filtered off using wattmann filter paper and washed twice with the chilled water. The isolated powder material was crystallized using a binary system (Methanol: DMF) to obtained an analytically pure product.

4.2.3 General procedure for the synthesis of 2-((5-(3-nitrophenyl)-1,3,4-oxadiazol-2-yl)methylthio)-5substitutedphenyl-1,3,4-oxadiazole (5a-5o).

In a THF (3 volume) containing RBF, 2-(chloromethyl)-5-(3-nitrophenyl)-1,3,4-oxadiazole (3) (0.01 mole) and various phenyl substituted 1,3,4-oxadiazole-2-thiol (4a-4o) (0.01 mole) were stirred at RT for 1.5-2.0 hrs using soft metal $\mathrm{CuI}(4 \mathrm{~mol} \%)$ catalysed $(0.02$ mole $)$ \& Triethylamine ( 1 eq. $)$ as a base catalyst. After completing the reaction [Monitored by TLC; Mobile phase: n-hexane: ethyl acetate $(8: 2 \mathrm{ml})]$, it was poured into crushed ice. The solid separated was isolated in dry powder by filtration and hot vacuum dryer. The product obtained was dried and recrystallized using ethanol.

The same method of synthesis was applied for all the derivatives. Various spectroscopic techniques were used to carry out the identification and characterization of derived products (5a-5o), i.e., IR, NMR $\left({ }^{1} \mathrm{H}\right.$ and $\left.{ }^{13} \mathrm{C}\right)$, elemental analysis, and MS.

\subsection{Analytical and Physical Data}

\subsubsection{2-(3-Nitrophenyl)-5-(((5-phenyl-1,3,4-oxadiazol-2-yl)thio)methyl)-1,3,4-oxadiazole. (5a)}

Yield: $87 \%$. mp $\left({ }^{0} \mathrm{C}\right)$ : 126. IR $\left(\mathrm{cm}^{-1}\right): 3078$ (C-H Aromatic stretching), 2931 (C-H Aliphatic stretching), 1638, 1578, 1456 (Aromatic ring skeleton), $1550 \& 1399$ (Aromatic $-\mathrm{NO}_{2}$ stretching), 1255 (C-O stretching), $780 \& 690$ (Aromatic m-disubstitution), 701 (C-S stretching).; ${ }^{1} \mathrm{H}$ NMR: (DMSO- $d_{6}$, $400 \mathrm{MHz}) \delta(\mathrm{ppm}): 8.69-8.67(\mathrm{~d}, 1 \mathrm{H}$, Aromatic proton), 8.57-8.56 (d, 1H, Aromatic proton), 8.50-8.45 (m, 2H, Aromatic proton), 8.01-7.99 (d,1H, Aromatic proton), 7.89-7.85 (m, 1H, Aromatic proton), 7.65-7.61 (m, 2H, Aromatic proton), 7.34-7.29 (m, 1H, Aromatic proton), 5.48 (s, 2H, Methylene group).; ${ }^{13} \mathrm{C}$ NMR: (DMSO-d6, 101.1 MHz) $\delta(\mathrm{ppm}): 165.16,162.20,159.39,153.29,149.04,133.71$, 131.57, 130.33, 129.80, 129.06, 127.08, 126.66, 122.67, 121.29, 24.46.; Mass (m/z): $381\left(\mathrm{M}^{+}\right)$.

\subsubsection{2-(3-Nitrophenyl)-5-(((5-(3-nitrophenyl)-1,3,4-oxadiazol-2-yl)methyl)thio)-1,3,4-oxadiazole. (5b)}

Yield: $81 \%$. mp $\left({ }^{0} \mathrm{C}\right): 225$. IR $\left(\mathrm{cm}^{-1}\right)$ : 3081 (C-H Aromatic stretching), 2945 (C-H Aliphatic stretching), 1621, 1588, 1442 (Aromatic ring skeleton), 1559 \& 1385 (Aromatic- $\mathrm{NO}_{2}$ stretching), 1259 (C-O stretching), $779 \& 693$ (Aromatic m-disubstitution), 698 (C-S stretching).; ${ }^{1} \mathrm{H}$ NMR: (DMSO- $d 6$, $400 \mathrm{MHz}) \delta(\mathrm{ppm}): 8.64-8.60(\mathrm{~d}, 1 \mathrm{H}$, Aromatic proton), 8.47-8.46 (d, 1H, Aromatic proton), 8.41-8.37 (d, 1H, Aromatic proton), 7.98-7.96 (d, 1H, Aromatic proton), 7.92-7.88 (m, 1H, Aromatic proton), 7.68-7.62 (m, 2H, Aromatic proton), 756-7.53 (m, 1H, Aromatic proton), 5.03 (s, 2H, Methylene group).; ${ }^{13} \mathrm{C}$ NMR: (DMSO-d6, 101.1 MHz) $\delta$ (ppm): 165.16, 162.20, 153.29, 149.11, 134.38, 133.71, 131.36, 130.33, 129.80, 127.60, 126.34, 122.67, 121.29, 24.46.. Mass (m/z): $426\left(\mathrm{M}^{+}\right)$.

\subsubsection{2-(4-Nitrophenyl)-5-(((5-(3-nitrophenyl)-1,3,4-oxadiazol-2-yl)methyl)thio)-1,3,4-oxadiazole. (5c)}

Yield: 93\%. mp $\left({ }^{0} \mathrm{C}\right)$ : 120. IR $\left(\mathrm{cm}^{-1}\right): 3075$ (C-H Aromatic stretching), 2953 (C-H Aliphatic stretching), 1648, 1583, 1450 (Aromatic ring skeleton), $1560 \& 1387$ (Aromatic $-\mathrm{NO}_{2}$ stretching), 1267 (C-O stretching), 868 (Aromatic p-disubstitution), 778 \& 694 (Aromatic m-disubstitution), 689 (C-S stretching).; ${ }^{1} \mathrm{H}$ NMR: (DMSO- $\left.d 6,400 \mathrm{MHz}\right) \delta(\mathrm{ppm})$ : 8.69-8.62(m, 1H, Aromatic proton), 8.51-8.48 (d, 1H, Aromatic proton), 8.28-8.27 (d, 1H, Aromatic proton), 7.99-7.95 (m, 2H, Aromatic proton), 7.82-7.77 (m, 2H, Aromatic proton), 7.51-7.45 (m, 1H, Aromatic proton), 5.09 (s, 2H, Methylene group).; ${ }^{13} \mathrm{C}$ NMR: (DMSO- $\left.d_{6}, 101.1 \mathrm{MHz}\right) \delta$ (ppm): 165.16, 162.20, 159.39, 153.29, 149.04, 147.39, 
$133.71,132.08,130.33,129.80,127.45,127.45,124.68,124.68,122.67,121.29,24.46 . ;$ Mass (m/z): $426\left(\mathrm{M}^{+}\right)$.

4.3.4 2-(2-Chlorophenyl)-5-(((5-(3-nitrophenyl)-1,3,4-oxadiazol-2-yl)methyl)thio)-1,3,4-oxadiazole. (5d)

Yield: $87 \%$ mp $\left({ }^{0} \mathrm{C}\right)$ : 112. IR $\left(\mathrm{cm}^{-1}\right)$ : 3025 (C-H Aromatic stretching), 2947 (C-H Aliphatic stretching), 1656, 1587, 1459 (Aromatic ring skeleton), $1585 \& 1380$ (Aromatic $-\mathrm{NO}_{2}$ stretching), 1260 (C-O stretching), 759 (Aromatic o-disubstitution), $786 \& 688$ (Aromatic m-disubstitution), 732 (C-Cl stretching), 696 (C-S stretching).; ${ }^{1} \mathrm{H}$ NMR: (DMSO- $\left.d_{6}, 400 \mathrm{MHz}\right) \delta(\mathrm{ppm}): 8.65-8.59$ (d, 2H, Aromatic proton), 8.47-8.38 (m, 3H, Aromatic proton), 7.92-7.88 (m, 3H, Aromatic proton), 5.06 (s, 2H, Methylene group).; ${ }^{13} \mathrm{C}$ NMR: (DMSO- $\left.d 6,101.1 \mathrm{MHz}\right) \delta(\mathrm{ppm}): 165.16,162.20,153.29,149.11$, 149.04, 134.38, 133.71, 131.36, 130.33, 129.80, 127.60, 126.34, 122.67, 121.29, 24.46. Mass (m/z): $415\left(\mathrm{M}^{+}\right)$.

4.3.5 2-(4-Chlorophenyl)-5-(((5-(3-nitrophenyl)-1,3,4-oxadiazol-2-yl)methyl)thio)-1,3,4-oxadiazole. (5e)

Yield: 92\%. mp $\left({ }^{0} \mathrm{C}\right)$ : 124. IR $\left(\mathrm{cm}^{-1}\right)$ : 3012 (C-H Aromatic stretching), 2958 (C-H Aliphatic stretching), 1663, 1578, 1444 (Aromatic ring skeleton), $1586 \& 1369$ (Aromatic-NO $\mathrm{NO}_{2}$ stretching), 1252 (C-O stretching), 872 (Aromatic p-disubstitution), $791 \& 696$ (Aromatic m-disubstitution), 732 (C-Cl stretching), 690 (C-S stretching).; ${ }^{1} \mathrm{H}$ NMR: (DMSO- $\left.d_{6}, 400 \mathrm{MHz}\right) \delta(\mathrm{ppm}): 8.78-8.76(\mathrm{~d}, 1 \mathrm{H}$, Aromatic proton), 8.59-8.51 (m, 2H, Aromatic proton), 7.90-7.83 (m, 2H, Aromatic proton), 7.61-7.55 (m, 3H, Aromatic proton), 5.05 (s, 2H, Methylene group).; ${ }^{13} \mathrm{C}$ NMR: (DMSO- $\left.d_{6}, 101.1 \mathrm{MHz}\right) \delta$ (ppm): 165.16, $162.20,159.39,153.29,149.04,137.31,133.71,130.33,129.80,129.46,129.46,128.39,128.09$, $128.09,122.67,121.29,24.46 .$. Mass $(\mathrm{m} / \mathrm{z}): 415\left(\mathrm{M}^{+}\right)$.

4.3.6 2-(2,4-Dichlorophenyl)-5-(((5-(3-nitrophenyl)-1,3,4-oxadiazol-2-yl)methyl)thio)-1,3,4-oxadiazole. (5f)

Yield: $82 \%$. mp $\left({ }^{0} \mathrm{C}\right): 130$. IR $\left(\mathrm{cm}^{-1}\right)$ : 3070 (C-H Aromatic stretching), 2956 (C-H Aliphatic stretching), 1684, 1549, 1458 (Aromatic ring skeleton), $1557 \& 1389$ (Aromatic- $\mathrm{NO}_{2}$ stretching), 1250 (C-O stretching), 861 (Aromatic p-disubstitution), 788 \& 691 (Aromatic m-disubstitution), 735 (C-Cl stretching), 758 (Aromatic o-disubstitution), 698 (C-S stretching).; ${ }^{1} \mathrm{H}$ NMR: (DMSO- $\left.d 6,400 \mathrm{MHz}\right)$ $\delta(\mathrm{ppm}): 8.32(\mathrm{~s}, 1 \mathrm{H}$, Aromatic proton), 8.03-8.02 (d, 1H, Aromatic proton), 7.63-7.62 $(\mathrm{d}, 1 \mathrm{H}$, Aromatic proton), 7.50-7.44 (m, 2H, Aromatic proton), 7.82-7.77 (m, 1H, Aromatic proton), 7.36-7.30 (m, 1H, Aromatic proton), 5.11 (s, 2H, Methylene group).; ${ }^{13} \mathrm{C}$ NMR: (DMSO- $\left.d 6,101.1 \mathrm{MHz}\right) \delta(\mathrm{ppm}): 165.16$, $162.20,153.29,149.11,149.04,135.87,134.93,133.71,131.73,130.33,129.80,128.52,128.48$, 127.88, 122.67, 121.29, 24.46. Mass (m/z): $448\left(\mathrm{M}^{+}\right)$.

\subsubsection{2-(2-Iodophenyl)-5-(((5-(3-nitrophenyl)-1,3,4-oxadiazol-2-yl)methyl)thio)-1,3,4-oxadiazole. (5g)}

Yield: $80 \%$. mp $\left({ }^{0} \mathrm{C}\right): 106$. IR $\left(\mathrm{cm}^{-1}\right): 3045$ (C-H Aromatic stretching), 2938 (C-H Aliphatic stretching), 1698, 1540, 1468 (Aromatic ring skeleton), $1598 \& 1382$ (Aromatic $-\mathrm{NO}_{2}$ stretching), 1258 (C-O stretching), $789 \& 689$ (Aromatic m-disubstitution), 768 (Aromatic o-disubstitution), 690 (C-S stretching), 561 (C-I stretching).; ${ }^{1} \mathrm{H}$ NMR: (DMSO- $\left.d 6,400 \mathrm{MHz}\right) \delta(\mathrm{ppm}): 8.56(\mathrm{~s}, 1 \mathrm{H}$, Aromatic proton), 8.29-8.28 (d, 1H, Aromatic proton), 8.10-8.09 (d, 1H, Aromatic proton), 7.98-7.95 (m, 2H, Aromatic proton), 7.80-7.75 (m, 2H, Aromatic proton), 7.09-7.04 (m, 1H, Aromatic proton), 4.98 (s, 2H, Methylene group).; ${ }^{13} \mathrm{C}$ NMR: (DMSO- $\left.d_{6}, 101.1 \mathrm{MHz}\right) \delta(\mathrm{ppm}): 165.16,162.20,153.29,149.17$, 149.04, 140.05, 136.13, 133.71, 131.96, 130.33, 129.80, 129.54, 126.04, 122.67, 121.29, 24.46. Mass $(\mathrm{m} / \mathrm{z}): 506\left(\mathrm{M}^{+}\right)$.

\subsubsection{2-(5-(((5-(3-Nitrophenyl)-1,3,4-oxadiazol-2-yl)methyl)thio)-1,3,4-oxadiazol-2-yl) phenol. (5h)}

Yield: 76\%. mp $\left({ }^{0} \mathrm{C}\right): 140$. IR ( $\left.\mathrm{cm}^{-1}\right): 3459$ (O-H stretching), 3058 (C-H Aromatic stretching), 2940 (C-H Aliphatic stretching), 1680, 1546, 1460 (Aromatic ring skeleton), 1591 \& 1378 (Aromatic $-\mathrm{NO}_{2}$ stretching), 1269 (C-O stretching), 781 \& 685 (Aromatic m-disubstitution), 769 (Aromatic odisubstitution), 697 (C-S stretching).; ${ }^{1} \mathrm{H}$ NMR: (DMSO-d6, $\left.400 \mathrm{MHz}\right) \delta(\mathrm{ppm}): 8.51$ (s, 1H, Aromatic proton), 8.25-8.24 (d, 1H, Aromatic proton), 7.94-7.93 (d, 1H, Aromatic proton), 7.67-7.61 (m, 1H, Aromatic proton), 7.40-7.33 (m, 1H, Aromatic proton), 7.19-7.12 (m, 1H, Aromatic proton), 6.98-6.91 
(m, 2H, Aromatic proton), 6.72 (s, 1H, -OH proton), 4.88 (s, 2H, Methylene group). ${ }^{13} \mathrm{C} \mathrm{NMR}$ : (DMSO$\left.d_{6}, 101.1 \mathrm{MHz}\right) \delta(\mathrm{ppm}): 165.16,162.20,158.44,153.29,150.08,149.04,133.71,131.79,130.33$, 130.12, 129.80, 122.67, 121.29, 119.86, 118.80, 111.70, 24.46. Mass (m/z): $397\left(\mathrm{M}^{+}\right)$.

\subsubsection{2-(3-Nitrophenyl)-5-(((5-(o-tolyl)-1,3,4-oxadiazol-2-yl)thio)methyl)-1,3,4-oxadiazole. (5i)}

Yield: $78 \%$. mp $\left({ }^{0} \mathrm{C}\right)$ : 80. IR $\left(\mathrm{cm}^{-1}\right): 3012$ (C-H Aromatic stretching), 2945 (C-H Aliphatic stretching), 1632, 1570, 1461 (Aromatic ring skeleton), 1548 \& 1386 (Aromatic $-\mathrm{NO}_{2}$ stretching), 1254 (C-O stretching), 778 \& 699 (Aromatic m-disubstitution), 759(Aromatic o-disubstitution), 712 (C-S stretching).; ${ }^{1} \mathrm{H}$ NMR: (DMSO- $\left.d_{6}, 400 \mathrm{MHz}\right) \delta(\mathrm{ppm}): 8.52$ (s, $1 \mathrm{H}$, Aromatic proton), 8.24-8.22 (d, $1 \mathrm{H}$, Aromatic proton), 7.94-7.92 (d, 1H, Aromatic proton), 7.67-7.62 (m, 2H, Aromatic proton), 7.31-7.28 (m, 3H, Aromatic proton), 4.89 (s, 2H, Methylene group), 2.59 (s, 3H, Methyl group). ${ }^{13} \mathrm{C}$ NMR: (DMSO- $d 6,101.1 \mathrm{MHz}) \delta(\mathrm{ppm}): 165.16,162.20,153.29,149.04,148.76,136.59,133.71,130.81$, 130.33, 129.80, 129.37, 128.75, 128.51, 126.53, 122.67, 121.29, 24.46, 20.24. Mass (m/z): $395\left(\mathrm{M}^{+}\right)$.

\subsubsection{2-(3-Nitrophenyl)-5-(((5-(p-tolyl)-1,3,4-oxadiazol-2-yl)thio)methyl)-1,3,4-oxadiazole. (5j)}

Yield: 85\%. mp $\left({ }^{0} \mathrm{C}\right)$ : 115. IR $\left(\mathrm{cm}^{-1}\right): 3055$ (C-H Aromatic stretching), 2926 (C-H Aliphatic stretching), 1630, 1549, 1455 (Aromatic ring skeleton), 1551 \& 1398 (Aromatic -NO2 stretching), 1245 (C-O stretching), 862 (Aromatic p-disubstitution), 788 \& 688 (Aromatic m-disubstitution), 702 (C-S stretching).; ${ }^{1} \mathrm{H}$ NMR: (DMSO- $\left.d_{6}, 400 \mathrm{MHz}\right) \delta(\mathrm{ppm}): 8.62$ (s, 1H, Aromatic proton), 8.25-8.23 (d, 1H, Aromatic proton), 7.94-7.92 (d, 1H, Aromatic proton), 7.67-7.52-7.46 (m, 2H, Aromatic proton), 7.29-7.23 (m, 2H, Aromatic proton), 4.95 (s, 2H, Methylene group), 2.39 (s, 3H, Methyl group).; ${ }^{13} \mathrm{C}$ NMR: (DMSO-d6, $\left.101.1 \mathrm{MHz}\right) \delta(\mathrm{ppm}): 165.16,162.20,159.39,153.29,149.04,140.60$, $133.71,130.33$, 129.80, 129.10, 129.10, 126.99, 126.99, 125.47, 122.67, 121.29, 24.46, 21.13. Mass (m/z): $395\left(\mathrm{M}^{+}\right)$.

\subsubsection{2-((5-(3-Nitrophenyl)-1,3,4-oxadiazol-2-yl)methylthio)-5-(4-bromophenyl)-1,3,4-oxadiazole. (5k)}

Yield: 89\%. mp $\left({ }^{0} \mathrm{C}\right): 202$. IR ( $\left.\mathrm{cm}^{-1}\right): 3056$ (C-H Aromatic stretching), 2921 (C-H Aliphatic stretching), 1656, 1575, 1457 (Aromatic ring skeleton), $1551 \& 1390$ (Aromatic $-\mathrm{NO}_{2}$ stretching), 1249 (C-O stretching), 870 (Aromatic p-disubstitution), $781 \& 693$ (Aromatic m-disubstitution), 715 (C-S stretching), 655 (C-Br stretching). ${ }^{1} \mathrm{H}$ NMR: (DMSO- $\left.d_{6}, 400 \mathrm{MHz}\right) \delta(\mathrm{ppm}): 8.43(\mathrm{~s}, 1 \mathrm{H}$, Aromatic proton), 8.10-8.08 (d, 1H, Aromatic proton), 7.98-7.96 (d, 1H, Aromatic proton), 7.65-7.59 (m, 2H, Aromatic proton), 7.59-7.46 (m, 1H, Aromatic proton), 7.26-7.21 (m, 1H, Aromatic proton), $4.78(\mathrm{~s}$, $2 \mathrm{H}$, Methylene group). ${ }^{13} \mathrm{C}$ NMR: (DMSO- $\left.d 6,101.1 \mathrm{MHz}\right) \delta(\mathrm{ppm}): 165.16,162.20,159.39,153.29$, 149.04, 133.71, 132.80, 132.80, 130.33, 130.11, 130.11, 129.80, 127.74, 125.31, 122.67, 121.29, 24.46. Mass (m/z): $460\left(\mathrm{M}^{+}\right)$.

\subsubsection{2-((5-(3-Nitrophenyl)-1,3,4-oxadiazol-2-yl)methylthio)-5-(3,4-dichlorophenyl)-1,3,4-oxadiazole. (5l)}

Yield: 84\%. mp $\left({ }^{0} \mathrm{C}\right): 220$. IR $\left(\mathrm{cm}^{-1}\right): 3011$ (C-H Aromatic stretching), 2951 (C-H Aliphatic stretching), 1658, 1548, 1450 (Aromatic ring skeleton), 1554 \& 1397 (Aromatic $-\mathrm{NO}_{2}$ stretching), 1251

(C-O stretching), 869 (Aromatic p-disubstitution), $788 \& 693$ (Aromatic m-disubstitution), 742 (C-Cl stretching), 705 (C-S stretching).; ${ }^{1} \mathrm{H}$ NMR: (DMSO-d6, $\left.400 \mathrm{MHz}\right) \delta(\mathrm{ppm}): 8.59(\mathrm{~s}, 1 \mathrm{H}$, Aromatic proton), 8.25-8.23 (d, 1H, Aromatic proton), 7.94-7.92 (d, 1H, Aromatic proton), 7.72-7.66 (m, 2H, Aromatic proton), $7.60(\mathrm{~s}, 1 \mathrm{H}$, Aromatic proton), 7.39-7.31 (m, 1H, Aromatic proton), $4.77(\mathrm{~s}, 2 \mathrm{H}$, Methylene group).; ${ }^{13} \mathrm{C}$ NMR: (DMSO- $\left.d_{6}, 101.1 \mathrm{MHz}\right) \delta(\mathrm{ppm}): 165.16,162.20,159.57,153.29$, $149.04,133.71,133.68,132.85,130.33,129.80,129.35,129.02,128.72,126.80,122.67,121.29$, 24.46.. Mass (m/z): $450\left(\mathrm{M}^{+}\right)$.

4.3.13 4-(5-((5-(3-Nitrophenyl)-1,3,4-oxadiazol-2-yl)methylthio)-1,3,4-oxadiazol-2-yl) benzene-1,2diol. $(5 \mathrm{~m})$

Yield: 85\%. mp $\left({ }^{0} \mathrm{C}\right): 185$. IR ( $\left.\mathrm{cm}^{-1}\right): 3489$ (O-H stretching), 3048 (C-H Aromatic stretching), 2935 (C-H Aliphatic stretching), 1632, 1579, 1461 (Aromatic ring skeleton), 1548 \& 1389 (Aromatic $-\mathrm{NO}_{2}$ 
stretching), 1250 (C-O stretching), 870 (Aromatic p-disubstitution), $779 \& 688$ (Aromatic mdisubstitution), 722 (C-S stretching).; ${ }^{1} \mathrm{H}$ NMR: (DMSO-d6, $\left.400 \mathrm{MHz}\right) \delta(\mathrm{ppm}): 8.58(\mathrm{~s}, 1 \mathrm{H}$, Aromatic proton), 8.22-8.20 (d, 1H, Aromatic proton), 7.92-7.90 (d, 1H, Aromatic proton), 7.67-7.60 (m, 1H, Aromatic proton), 7.07-7.01 (m, 1H, Aromatic proton), 6.94-6.85 (m, 2H, Aromatic proton), 4.93 (s, $2 \mathrm{H}$, Methylene group), 3.34 (s, $1 \mathrm{H}, p-\mathrm{OH}$ proton), 2.90 (s, $1 \mathrm{H}, o-\mathrm{OH}$ proton). ${ }^{13} \mathrm{C}$ NMR: (DMSO- $d_{6}$, $101.1 \mathrm{MHz}) \delta(\mathrm{ppm}): 165.16,162.20,159.57,153.29,149.04,148.26,148.22,133.71,130.33,129.80$, $122.67,121.45,121.29,119.07,117.60,112.31,24.46$. Mass $(\mathrm{m} / \mathrm{z}): 413\left(\mathrm{M}^{+}\right)$.

\subsubsection{2-((5-(3-Nitrophenyl)-1,3,4-oxadiazol-2-yl)methylthio)-5-(4-(difluoromethyl)phenyl)-1,3,4-oxadiazole.} $(5 n)$

Yield: 91\%. mp $\left({ }^{0} \mathrm{C}\right)$ : 195. IR $\left(\mathrm{cm}^{-1}\right)$ : 3052 (C-H Aromatic stretching), 2932 (C-H Aliphatic stretching), 1658, 1588, 1454 (Aromatic ring skeleton), 1555 \& 1392 (Aromatic $-\mathrm{NO}_{2}$ stretching), 1259 (C-O stretching), 1089 (C-F stretching), 863 (Aromatic p-disubstitution), 784 \& 687 (Aromatic mdisubstitution), 698 (C-S stretching).; ${ }^{1} \mathrm{H}$ NMR: (DMSO- $\left.d 6,400 \mathrm{MHz}\right) \delta(\mathrm{ppm}): 8.61$ (s, 1H, Aromatic proton), 8.09-8.07 (d, 1H, Aromatic proton), 7.90-7.88 (d, 1H, Aromatic proton), 7.65-7.57 (m, 3H, Aromatic proton), 7.51-7.44 (m, 2H, Aromatic proton), 6.40-6.37 (t, 1H, Methine proton), 5.01 (s, 2H, Methylene group). ${ }^{13} \mathrm{C}$ NMR: (DMSO- $\left.d_{6}, 101.1 \mathrm{MHz}\right) \delta(\mathrm{ppm}): 165.16,162.20,159.39,153.29$, $149.04,140.00,133.71,131.71,130.33,129.80,126.36,126.36,125.44,125.44,122.67,121.29$, 116.13, 24.46. Mass (m/z): $431\left(\mathrm{M}^{+}\right)$.

\subsubsection{2-((5-(3-Nitrophenyl)-1,3,4-oxadiazol-2-yl)methylthio)-5-(2,4-dinitrophenyl)-1,3,4-oxadiazole. (5o)}

Yield: $79 \%$. mp $\left({ }^{0} \mathrm{C}\right)$ : 188. IR $\left(\mathrm{cm}^{-1}\right)$ : 3027 (C-H Aromatic stretching), 2989 (C-H Aliphatic stretching), 1634, 1579, 1454 (Aromatic ring skeleton), 1560 \& 1398 (Aromatic $-\mathrm{NO}_{2}$ stretching), 1258 (C-O stretching), $789 \& 692$ (Aromatic m-disubstitution), 765 (Aromatic o-disubstitution), 734 (C-S stretching).; ${ }^{1} \mathrm{H}$ NMR: (DMSO- $\left.d_{6}, 400 \mathrm{MHz}\right) \delta(\mathrm{ppm}): 9.12$ (s, $1 \mathrm{H}$, Aromatic proton of between two nitro group), 8.66-8.64 (d, 1H, Aromatic proton), 8.51 (s, $1 \mathrm{H}$, Aromatic proton), 8.25-8.23 (d, $1 \mathrm{H}$, Aromatic proton), 8.06-8.04 (d, 1H, Aromatic proton), 7.95-7.89 (m, 1H, Aromatic proton), 7.67-7.60 (m, 1H, Aromatic proton), 4.83 (s, 2H, Methylene group).; ${ }^{13} \mathrm{C}$ NMR: (DMSO-d6, $\left.101.1 \mathrm{MHz}\right) \delta(\mathrm{ppm})$ : $165.16,162.20,153.29,150.56,149.04,147.61,146.01,133.71,131.65,130.33,130.14,129.80$, 126.87, 122.67, 121.45, 121.29, 24.46. Mass (m/z): $471\left(\mathrm{M}^{+}\right)$.

\section{References}

1. Adil A., Othman M., and Sarah A. (2019) 1,3,4-Oxadiazole, 1,3,4-thiadiazole and 1,2,4-triazole derivatives as potential anti-bacterial agents. Arab. J. Chem., 12 (7) 1660-1675.

2. (a) Yang S. J., Lee S. H., Kwak H. J., and Gong Y. D. (2013) Regio selective Synthesis of 2-AminoSubstituted 1,3,4-Oxadiazole and 1, 3, 4-Thiadiazole Derivatives via Reagent-Based Cyclization of Thiosemicarbazide Intermediate. J. Org. Chem., 78 438-444. (b) Prezent M.A., and Baranin S.V. (2019) A convenient route to the 1,2,5-oxadiazole-substituted 1,2,4-triazolo[1,5-a]pyrimidine derivatives. Chem Heterocycl Comp., 55 1131-1134.

3. Badran M., Moneer A., Rafaat H., and El-Malah A. (2007) Synthesis and Anti-microbial Activity of Novel Quinoxaline Derivatives. J. Chin. Chem. Soc., 54 (2) 469-478.

4. Plech T., Wujec M., Siwek A., Kosikowska U., and Malm A. (2013) The effect of C-5 substituent on antibacterial activity. Med Chem Res., 222531.

5. Sun X., Hong Z., Liu M., Guo S., Yang D., Wang Y., Lan T., Gao L., Qi H., and Gong P. (2017) Design, synthesis, and biological activity of novel tetrahydropyrazolopyridone derivatives as FXa inhibitors with potent anticoagulant activity. Bioorg. Med. Chem., 25 (10) 2800-2810.

6. Mickevičius V., Vaickelionienè R., and Sapijanskaite B. (2009) Synthesis of substituted 1,3,4-oxadiazole derivatives. Chem Heterocycl Comp., 45 215-218.

7. Stephens C., Tanious F., Kim, S., Wilson W., Schell W., Perfect J., Franzblau S., and Boykin D. (2001) Diguanidino and "Reversed" Diamidino 2,5-Diarylfurans as Antimicrobial Agents. J. Med. Chem., 44 (11) 1741-1748.

8. (a) Abd El-Meguid E., Awad H., and Anwar M. (2019) Synthesis of New 1,3,4-Oxadiazole-benzimidazole Derivatives as Potential Antioxidants and Breast Cancer Inhibitors with Apoptosis Inducing Activity. Russ. 
J. Gen. Chem., 89, 348-356. (b) Mohi El-Deen E., Mohamed N.A., El-Serwy W., Abdelghany T., and Nossier E., (2016) Synthesis, molecular docking and cytotoxicity evaluation of novel 1,2-disubstituted benzimidazole derivatives against liver and breast cancer cell lines. Res J Pharm Biol Chem Sci., 7, 15991615.

9. Singh A., Lohani M., and Parthsarthy R. (2013) Synthesis, Characterization and Anti-Inflammatory Activity of Some 1, 3,4 -Oxadiazole Derivatives. Iran. J. Pharm. Res., 12 (2) 319-323.

10. Zheng X., Li Z., Wang Y., Chen W., Huang Q., Liu C., and Song G. (2003) Synthesis and insecticidal activities of novel 2,5-disubstituted-1,3,4-oxadiazoles. J. Fluorine Chem., 123 163-169.

11. Almasirad A., Tabatabai S., Faizi M., Kebriaeezadeh A., Mehrabi N., Dalvandi A., and Shafiee, A. (2004) Synthesis and anticonvulsant activity of new 2-substituted-5- [2-(2-fluorophenoxy)phenyl]-1,3,4oxadiazoles and 1,2,4-triazoles. Bioorg. Med.Chem. Lett., 24 6057-6059.

12. Mochona B., Qi X., Euynni S., Sikazwi D., Mateeva N., and Soliman K. (2016) Design and Evaluation of Novel Oxadiazole Derivatives as Potential Prostate Cancer Agents. Bioorg. Med.Chem. Lett., 26 (12) $2847-$ 2851.

13. Kadi A., El-Brollosy N., Al-Deeb O., Habib E., Ibrahim T., and El-Emam A. (2007) Synthesis, antimicrobial, and anti-inflammatory activities of novel 2-(1-adamantyl)-5-substituted-1,3,4-oxadiazoles and 2(1-adamantylamino)-5-substituted-1,3,4-thiadiazoles. Eur. J. Med. Chem., 42 235-242.

14. Ramazani A., and Souldozi A. (2008) Iminophosphorane-mediated one-pot synthesis of 1,3,4-oxadiazole derivatives. Arkivoc, 16 235-242.

15. Ramazani A., Ahmadi Y., and Tarasi R. (2011) Efficient One-Pot Synthesis of Disubstituted 1,3,4Oxadiazole Derivatives from the Reaction of (N-Isocyanimino)triphenylphosphorane, Acetaldehyde, a Secondary Amine, and an Electron-Poor (E)-Cinnamic Acid. Heteroat. Chem., 22 (1) 79-84.

16. (a) Lu J., Jiang F., Lu A., and Zhang G. (2016) Linkers Having a Crucial Role in Antibody-Drug Conjugates. Int J Mol Sci., 17 561. (b) Krishna, C., Bhargavi, M., and Krupadanam, G. (2018) Design, Synthesis, and Cytotoxicity of Semisynthetic Betulinic Acid-1,2,4-Oxadiazole Amide Derivatives. Russ. J. Gen. Chem., 88 312-318.

17. Zhao T., Zhao Z., and Lu F. (2020) Two- and three-dimensional QSAR studies on hURAT1 inhibitors with flexible linkers: topomer CoMFA and HQSAR. Mol Divers., 24 141-154.

18. Cramer R., Patterson D., and Bunce J. (1988) Comparative molecular field analysis (CoMFA). 1. Effect of shape on binding of steroids to carrier proteins. J Am Chem Soc., 110 (18) 5959-5967.

19. Rayam P., Polkam N., Kummari B., Banothu V., Gandamalla D., Reddy N., and Anireddy J. (2019) Synthesis and Biological Evaluation of New Ibuprofen-1,3,4-oxadiazole-1,2,3-triazole Hybrids. $J$. Heterocycl. Chem., 56 (1) 296-305.

20. Jasiński, R. (2015) On the question of zwitterionic intermediates in 1,3-dipolar cycloadditions between hexafluoroacetone and sterically crowded diazocompounds. J. Fluor. Chem., 176 35-39.

21. Iyer, V., Gurupadayya B., Koganti V., Inturi B., and Chandan R. (2017) Design, synthesis and biological evaluation of 1,3,4-oxadiazoles as promising anti-inflammatory agents. Med Chem Res., 26 190-204.

22. Muhi-eldeen Z., Juma'a G., Al-kaissi, G., and Nouri L. (2008) Anti-microbial activity of some new oxadiazole derivatives. Jordan J. Chem., 3 (3) 233-243.

23. Wang S., Wang K., Kong X., Zhang S., Jiang G., Ji F. (2019) DMF as Methine Source: Copper-Catalyzed Direct Annulation of Hydrazides to 1,3,4-Oxadiazoles. Adv. Synth. Catal., 361 (17) 3986-3990.

24. de Oliveira C., Lira B., Barbosa-Filho J., Lorenzo J., and de Athayde-Filho P. (2012) Synthetic approaches and pharmacological activity of 1,3,4-oxadiazoles: a review of the literature from 2000-2012. Molecules, 17 (9) 10192-10231.

25. Kapadiya K., Kotadiya R., Kavadia K., Kothari R., Mehariya K., and Khunt R. (2015) Synthesis and Microbial Evaluation of Versatile Base Catalyzed Chiral Tetrahydrobenzofuran Derivatives via Multicomponent Reaction. Lett Drug Des Discov., 13 (6) 505-513.

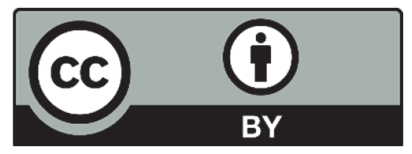

(C) 2021 by the authors; licensee Growing Science, Canada. This is an open access article distributed under the terms and conditions of the Creative Commons Attribution (CC-BY) license (http://creativecommons.org/licenses/by/4.0/). 